Published in final edited form as:

Ann Behav Med. 2015 October ; 49(5): 776-781. doi:10.1007/s12160-015-9699-y.

\title{
The Strength of Family Ties: Perceptions of Network Relationship Quality and Levels of C-Reactive Proteins in the North Texas Heart Study
}

\author{
Bert N. Uchino, Ph.D., \\ Department of Psychology, University of Utah, Salt Lake City, UT, USA \\ John M. Ruiz, Ph.D., \\ Department of Psychology, University of North Texas, Denton, TX, USA \\ Timothy W. Smith, Ph.D., \\ Department of Psychology, University of Utah, Salt Lake City, UT, USA
}

Joshua M. Smyth, Ph.D.,

Department of Psychology, Pennsylvania State University, University Park, State College, PA, USA

\section{Daniel J. Taylor, Ph.D.,} Department of Psychology, University of North Texas, Denton, TX, USA

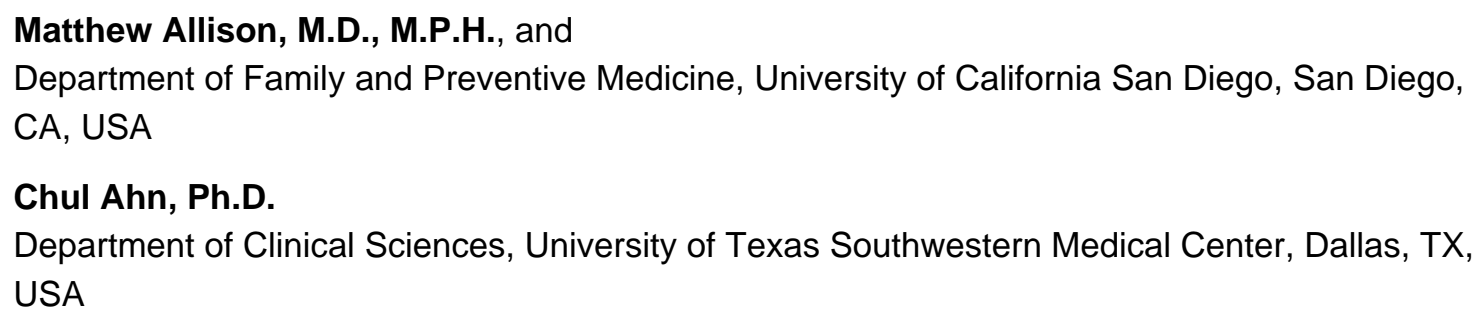

\section{Abstract}

Background-Although the quality of one's social relationships has been linked to important physical health outcomes, less work has been conducted examining family and friends that differ in their underlying positivity and negativity.

Purpose-The main aim of this study was to examine the association between supportive, aversive, and ambivalent family/friends with levels of C-reactive proteins.

Methods-Three hundred participants from the North Texas Heart Study completed the social relationships index and a blood draw to assess high-sensitivity C-reactive proteins (hs-CRPs).

Correspondence to: Bert N. Uchino, bert . uchino@psych. utah.edu.

Authors' Statement of Conflict of Interest and Adherence to Ethical Standards Authors Uchino, Ruiz, Smith, Smyth, Taylor, Allison, and Chul have no conflict of interest. All procedures, including the informed consent process, were conducted in accordance with the ethical standards of the responsible committee on human experimentation (institutional and national) and with the Helsinki Declaration of 1975 , as revised in 2000 . 
Results-After standard controls, the number of supportive family members predicted lower hsCRP levels, whereas the number of ambivalent family members predicted higher hs-CRP levels. These links were independent of depressive symptoms and perceived stress.

Conclusions-These data highlight the importance of considering specific types of relationships and their underlying positive and negative aspects in research on social ties and physical health.

The quality of one's social relationships has been reliably related to both morbidity and mortality $[1,2]$. Most prior research in this area has focused on the health benefits of social support. [1] However, relationship positivity represents only one important dimension of relationships. A small, but growing literature indicates that relationship negativity is related to worse health outcomes [3]. As a result, a more comprehensive approach to understanding the health consequences of relationships would be to consider both positive and negative aspects [4]. Such a broad framework makes salient relationships that differ in their underlying positivity/negativity including supportive (i.e., high positivity and no negativity), aversive (i.e., no positivity and high negativity), and ambivalent (i.e., high positivity and high negativity) social ties.

The specification of ambivalent ties is particularly interesting because much less is known about its potential links to health-relevant biological outcomes. Such relationships appear to reflect a history of "mixed" interpersonal transactions or interpersonal tensions in the context of a previously positive relationship. For instance, adult children's current emotional and behavioral problems were related to greater parental perceptions of ambivalence toward the adult child [5]. Importantly, many relationships are characterized by both positive and negative qualities [4]. Up to $50 \%$ of important network members are viewed as sources of ambivalence [4]. These data suggest that perceptions of ambivalence toward relationships are common and hence have ample opportunity to influence health outcomes. Consistent with this suggestion, research suggests that despite the positivity associated with ambivalent ties, the co-occurring negativity is uniquely related to adverse biological outcomes in both laboratory and daily life assessments [6, 7]. In one study of 150 married older couples, it was found that when partners viewed each other as a source of ambivalence, that was related to greater coronary artery calcium plaque burden [7].

One other unexplored direction in testing this broad model is the specific social ties that matter most within these relationship categories (i.e., ambivalent, supportive, and aversive). More generally, less work has examined the relative contributions of specific types of relationships such as family or friends to the relationships and health link. An examination of specific relationships is important because family members often serve broader functions compared to friends, including emotional support and help with activities of daily living [8]. However, family members also tend to be relatively greater sources of negative support transactions which may create strain in relationships (e.g., unwanted advice and modeling poor behaviors) [9].

If family members provide broader support functions but are also greater sources of negativity, then they may be particularly important contributors to health. Consistent with this possibility, Shor and colleagues [2] found that support from family members was a stronger predictor of lower mortality compared to support from friends. Moreover, it is 
possible that family members that are sources of both positive and negative interactions (i.e., ambivalence) might be linked to worse health outcomes. Such relationships may be particularly impactful given their unpredictability, proximity, and influence which might serve as chronic sources of stress [4].

The major aims of this study were thus to examine the association between specific network members (i.e., family and friends) and high-sensitivity C-reactive protein (hs-CRP) using a comprehensive assessment of positive and negative social ties (i.e., supportive, aversive, and ambivalent). Hs-CRP was examined because it is a well-documented inflammatory marker of future health problems including cardiovascular disease, diabetes, and cancer [10-12]. In fact, CRP appears to play a role in the inflammation that is a part of the atherogenic process $[13,14]$. In this regard, prior work has linked positivity or negativity in relationships to inflammation $[15,16]$. However, this is the first study to test if perceptions of ambivalence toward family members versus friends at the social network level predict inflammation. Consistent with prior work on the potential importance of family ties, it was predicted that supportive and ambivalent family ties would be more consistently related to lower and higher hs-CRP levels, respectively, compared to supportive and ambivalent friends.

\section{Method}

\section{Participants}

The North Texas Heart Project is a community sample of 300 adults ( 150 men, 150 women), ages 21 to 70 years. The sample is stratified by age within gender and race/ethnicity. The mean age at enrollment was 42.44 years $(S D=12.76)$, with men younger than women $(M=41$ vs. $44, p=.03)$. The diverse sample includes $60 \%$ non-Hispanic Whites, $15 \%$ non-Hispanic Blacks, and $19 \%$ Hispanic/Latino of which $75 \%$ self-identified as being of Mexican descent. A majority of participants were married (60\%), own a home $(63 \%)$, and are employed outside the home (79\%). Participants represented a broad range of educational backgrounds although more than $86 \%$ reported some college. Similarly, the sample reflects significant income diversity with $12 \%$ reporting a household income less than $\$ 20,000$ and $10 \%$ above $\$ 150,000$, and the modal annual household income was reported to be $\$ 75,000$ to $\$ 100,000$.

\section{Procedures}

All sessions were conducted on Thursday mornings at a single-site vascular medicine clinic. Following arrival and consent, all participants underwent a brief physical exam, personal and family medical history, and a review of current medications and conditions. Participants were rescheduled if there was evidence of acute illness/infection. A fasting blood draw was used to assess levels of hs-CRP. Finally, participants completed a psychosocial survey which included the Social Relationship Index, Center for Epidemiologic Studies (CES) Depression scale, and Perceived Stress Scale (see below).

\section{Measures}

Social Relationships Index-The Social Relationships Index (SRI)-short form instructs individuals to list the initials of individuals in the following domains: (a) spouse/significant 
other, (b) other family, and (c) friends. The categories of other family and friends were limited to three people in order to keep completion of the SRI to a manageable time frame. These network members were then rated in terms of how helpful and upsetting they are $(1=$ not at all, $6=$ extremely) when the participant needs emotional, tangible, or informational support (see [17] for psychometric information). In order to reduce participant demand, participants rated network members across these support components (as one question) given that evidence suggests that these components are highly correlated [17]. Based on prior work, different categories of social relationships were operationalized as the total number of individuals in one's network who were sources of support (i.e., "2" or greater on positivity and only a "1" on negativity), aversion (i.e., only a "1" on positivity and "2" or greater on negativity), or ambivalence (i.e., "2" or greater on both positivity and negativity) [4]. Indifferent ties were not examined as this short form did not include an assessment of social acquaintances. Based on the definition of families by the US Census, the spouse and other family categories were combined to produce an overall index of family support, ambivalence, or aversion.

Center for Epidemiologic Studies Depression Scale-The Center for Epidemiologic Studies Depression (CES-D) is a 20-item scale that assesses depressive symptoms. The Cronbach's alpha was high in both patient and control samples (.90), with a 4-week test-retest correlation of .67 [18]. The internal consistency of the CES-D in the current study was similarly high (.80).

Perceived Stress Scale-The Perceived Stress Scale (PSS) contains 10 items and measures general perceptions of stress. The Cronbach's alpha for the PSS has been reported at .75 [19]. The internal consistency of the scale for our study was similarly high (.88).

High-sensitivity CRP-The serum samples were assayed in duplicate using a Human CRP (hs) ELISA assay kit (BC-1119) from BioCheck (Foster City, CA). The assay used 5 $\mathrm{uL}(\times 100$ dil) of sample per well and had a standard curve range of 0.005 to $0.1 \mathrm{mg} / \mathrm{L}$. The lower limit of detection of the assay is $0.1 \mathrm{mg} / \mathrm{L}$. Assay precision as determined by interassay and intra-assay coefficients of variation (\%CV) was 3.3 and $4.4 \%$, respectively. Accuracy of the assay was determined by the linearity of dilution which yielded a correlation coefficient of 0.99 .

\section{Data Management and Statistical Analyses Plan}

Inspection of hs-CRP values revealed 12 participants with levels over 10. Consistent with prior work and recommendations by the Center for Disease Control and Prevention/ American Heart Association [20], these individuals were deleted as they likely reflect acute inflammatory reactions and not chronic inflammation. Three additional participants who had missing data for the relevant variables in the model were deleted ( 1 for body mass, 2 for hsCRP, final $n=285$ ). CRP was natural log-transformed to normalize the distribution prior to analyses. For primary analyses, simultaneous regression analyses with predictors centered at the grand mean were used to examine the links between each individual social network category and hs-CRP levels. All analyses controlled for age, ethnicity (contrast coded), gender, body mass, and medication use (i.e., no or yes for hypertension, lipids, other cardiac, 
and diabetes [21]). Secondary analyses also examined the relative prediction of hs-CRP by the family and friendship relationship categories via a simultaneous regression model. Finally, we examined if these relationship quality factors continued to predict CRP after considering the role of depression and perceived stress.

\section{Results}

\section{Sample Characteristics}

The mean level of hs-CRP for this sample was $2.66(S D=2.50)$ and the mean body mass index was $29.0(S D=6.3) ; 15 \%$ were taking a blood pressure medication, $14 \%$ a lipid medication, $5.6 \%$ other cardiac medications, and $3.9 \%$ a diabetes medication. Examination of familial ties showed that perceptions of ambivalent ties were most common $(M=2.10$, $S D=1.32)$, followed closely by perceptions of supportive ties $(M=1.25, S D=1.24)$, with a small number of aversive family ties $(M=.13, S D=.40)$. In comparison, the friendship category had the highest number of perceived supportive ties $(M=1.48, S D=1.16)$, closely followed by perceived ambivalent ties $(M=.97, S D=1.08)$, and a small number of perceived aversive friendship ties $(M=.02, S D=.19)$. Although a short version of the SRI was used to reduce demand on participants, these relative network proportions are comparable to our prior work using the full SRI [17]. Please see Table 1 for zero-order correlations among major study variables.

\section{Relationships and CRP}

Consistent with predictions, family members were the most reliable predictors of inflammation. As shown in Tables 2 and 3, the number of supportive family ties was associated with lower hs-CRP ( $b=-.10,95 \%$ confidence interval $(\mathrm{CI})[-.19,-.01], \beta=-.11$, $p=.029)$, whereas the number of ambivalent family ties predicted higher hs-CRP ( $b=.08,95$ $\%$ CI $[.0005, .17], \beta=.10, p=.049)$. The number of aversive family ties did not significantly predict hs-CRP levels ( $b=.21,95 \% \mathrm{CI}[-.07, .49], \beta=.07, p=.14)$. In comparison, friends were not significant predictors of inflammation. Neither the number of supportive $(b=-.02$, $95 \%$ CI $[-.12, .07], \beta=-.02, p=.63)$, ambivalent $(b=.04,95 \%$ CI $[-.06, .15], \beta=.04, p=.41)$, nor aversive $(b=-.11,95 \% \mathrm{CI}[-.69, .47], \beta=-.02, p=.71)$ friends was significantly associated with hs-CRP levels.

Secondary analyses focused on the unique prediction of hs-CRP between family and friends by including both in the same model given they were correlated. In the model contrasting the number of supportive family and friends, the number of supportive family members continued to be a predictor of inflammation ( $b=-.10,95 \%$ CI $[-.20,-.01], \beta=-.12, p=.03)$, whereas the number of supportive friends was not $(p=.80)$. Although attenuated slightly, the number of ambivalent family ties was still marginally associated with higher hs-CRP ( $b=.08$, $95 \%$ CI $[-.006, .17], \beta=.10, p=.069)$ in comparison to the number of ambivalent friends ( $p=$. 76). Finally, ancillary analyses were run to determine if family support and ambivalence continued to predict hs-CRP when statistically controlling for the individual factors of depression and perceived stress. Although depression and perceived stress did not predict hs-CRP levels, the number of supportive family ties $(p=.02)$ and ambivalent family ties $(p=$. 03) continued to predict hs-CRP levels. 


\section{Discussion}

The main aim of this study was to examine links between family and friendships that differed in their underlying positivity/negativity and CRP levels. As predicted, the number of supportive family members was related to lower hs-CRP and the number of ambivalent family members was related to higher hs-CRP, but this association was not found for the number of supportive and ambivalent friends. Importantly, hs-CRP is a well-documented inflammatory marker as it predicts a number of future health problems including cardiovascular disease, metabolic disorders, cancer, and frailty [10-12].

This appears to be the first study examining the association between these family and friend social network categories and CRP levels. Most prior work in this area has focused on links between global positive aspects of relationships (e.g., social support) and inflammation [1]. However, global and specific relationship assessments are separable as family and friends differentially contribute to support resources and strain [8, 9]. It has been argued that family members are more important than friends due to their general availability, norm of support, and closeness $[8,9]$. Family members are also sources of greater negative interactions compared to friends suggesting that they also have the potential to have detrimental influences on health [9]. Consistent with this possibility, the number of supportive and ambivalent familial ties predicted CRP levels whereas the number of supportive and ambivalent friendships did not. Although we did not find the number of aversive family members to predict CRP, it has been argued that aversive ties are less impactful because of the lack of positivity in these ties. Thus, individuals do not care as much about aversive ties which minimizes their impact [4].

Although researchers have argued for the strength of family members, some prior work suggests that friends are also important contributors to health and well-being [22]. So why was stronger evidence not found for potential links between the number of supportive and ambivalent friends and inflammation? There are several potential reasons that will require further examination. First of all, this study utilized a brief form of the SRI. It is possible that a more comprehensive assessment of friendships might reveal stronger evidence for their links to health $[4,22]$. It is also possible that links between friendships and health outcomes might be more evident in younger samples in which peers appear to be important as sources of support. [22] Future work that continues to contrast different relationships (including coworkers) while addressing these issues would be important to resolve this issue.

The exact mechanisms responsible for these links between family ties and CRP will also require future work. Such mechanisms could be as simple as increased contact, availability, and closeness [8]. These interpersonal processes could increase the frequency and efficacy of support transactions and hence might be related to health benefits. Likewise, ambivalence in such relationships can lead to increased interpersonal stress and rumination that could adversely influence health [4]. More complicated mechanisms include links to selfregulatory processes. For instance, social support has been linked to greater self-regulatory capacity due to the availability of important social resources which may be health promoting [23]. In comparison, perceptions of ambivalence are linked to less support seeking and poorer quality received support. [22] As a result, the lack of access to support, or exposure 
to negative support transactions, may deplete an individual by requiring the expenditure of more personal resources when coping with stress [23]. Future research testing selfregulatory resources as a direct mediator would inform this issue.

There are several important limitations of this study. First, the study is cross sectional so strong causal inferences cannot be made until longitudinal work is conducted. In a related point, although hs-CRP is related to the development and course of health problems, longitudinal studies will again be needed to examine if such links underlie the health risks and buffers associated with ambivalent and supportive family ties. Nevertheless, this is one of the first studies testing the association between these categories of social ties and CRP and suggests the promise of future investigations incorporating relationship-specific approaches.

\section{Acknowledgments}

This research was generously supported by grant number R01 HL109340 from the National Heart, Lung, and Blood Institute and the Basic Behavioral and Social Sciences Research Opportunity Network (OppNet), National Institutes of Health (PI: John M. Ruiz).

\section{References}

1. Holt-Lunstad J, Smith TB, Layton B. Social relationships and mortality: A meta-analysis. PLoS Med. 2010; 7:1-20.10.1371/journal.pmed.1000316

2. Shor E, Roelfs DJ, Yogev T. The strength of family ties: A meta-analysis and meta-regression of self-reported social support and mortality. Soc Networks. 2013; 35:626-638.

3. Brooks K, Dunkel SC. Social negativity and health. Soc Personal Psychol Compass. 2011; 5:904918.

4. Uchino BN, Holt-Lunstad J, Uno D, et al. Heterogeneity in the social networks of young and older adults: Prediction of mental health and cardiovascular reactivity during acute stress. J Behav Med. 2001; 24:361-382. [PubMed: 11523333]

5. Birditt KS, Miller LM, Fingerman KL, et al. Tensions in the parents and adult child relationship: Links to solidarity and ambivalence. Psychol Aging. 2009; 24:287-295.10.1037/a0015196 [PubMed: 19485648]

6. Holt-Lunstad J, Uchino BN, Smith TW, et al. Social relationships and ambulatory blood pressure: Structural and qualitative predictors of cardiovascular function during everyday social interactions. Health Psychol. 2003; 22:388-397. [PubMed: 12940395]

7. Uchino BN, Smith TW, Berg CA. Spousal relationship quality and cardiovascular risk: Dyadic perceptions of relationship ambivalence are associated with coronary calcification. Psychol Sci. 2014; 25:1037-1042. [PubMed: 24501110]

8. Rook, KS.; Shuster, TL. Compensatory processes in the social networks of older adults. In: Pierce, G.; Sarason, B.; Sarason, I., editors. The handbook of social support and the family. NY: Plenum; 1990. p. 219-248.

9. Gallant MP, Spitze GD, Prohaska TR. Help of hindrance? How family and friends influence chronic illness self-management among older adults. Res Aging. 2007; 29:375-409.

10. Guo Y, Pan L, Du C, et al. Association between C-reactive protein and risk of cancer: A metaanalysis of prospective cohort studies. Asian Pac J Cancer Prev. 2013; 14:243-248. [PubMed: 23534731]

11. Lee CC, Adler AI, Sandhu MS, et al. Association of C-reactive protein with type 2 diabetes: Prospective analysis and meta-analysis. Diabetologia. 2009; 52:1040-1047. [PubMed: 19326095]

12. Libby P. Inflammation in atherosclerosis. Nature. 2002; 420:868-874.10.1161/hc0902.104353 [PubMed: 12490960] 
13. Liuzzo G, Santamaria M, Biasucci LM, et al. Persistent activation of nuclear factor kappa-B signaling pathway in patients with unstable angina and elevated levels of C-reactive protein. J Am Coll Card. 2007; 49:185-194.

14. Verma S, Szmitko PE, Yeh ETH. C-reactive protein: Structure affects function. Circ. 2004; 109:1914-1917.

15. Friedman EM, Hayney MS, Love GD, et al. Social relationships, sleep quality, and interleukin-6 in aging women. Proc Natl Acad Sci U S A. 2005; 102:18757-18762. [PubMed: 16339311]

16. Yang YC, Schorpp K, Harris KM. Social support, social strain, and inflammation: Evidence from a national longitudinal study of U.S. adults. Soc Sci Med. 2014; 107:124-135. [PubMed: 24607674]

17. Campo RA, Uchino BN, Holt-Lunstad J, et al. The assessment of positivity and negativity in social networks: The reliability and validity of the Social Relationships Index. J Community Psychol. 2009; 37:471-486.10.1002/jcop.20308

18. Radloff LS. The CES-D scale: A self-report depression scale for research in the general population. Appl Psychol Meas. 1977; 1:385-401.

19. Cohen, S.; Williamson, GM. Perceived stress in a probability sample in the United States. In: Spacapan, S.; Oskamp, S., editors. The social psychology of health. London: Sage; 1987. p. 31-67.

20. Pearson TA, Mensah GA, Alexander RW, et al. Markers of inflammation and cardiovascular disease: Application to clinical and public health practice: A statement for healthcare professionals from the centers for disease control and prevention and the American Heart Association. Circulation. 2003; 107:499-511. [PubMed: 12551878]

21. O'Connor MF, Bower JE, Cho HJ, et al. To assess, to control, to exclude: Effects of biobehavioral factors on circulating inflammatory markers. Brain Behav Immun. 2009; 23:887-897.10.1016/ j.bbi.2009.04.005 [PubMed: 19389469]

22. Holt-Lunstad JL, Uchino BN, Smith TW, et al. On the Importance of Relationship Quality: The Impact of Ambivalence in Friendships on Cardiovascular Functioning. Ann Behav Med. 2007; 33:278-290. [PubMed: 17600455]

23. Lane B, Coan JA. Social baseline theory: The role of social proximity in emotion and economy of action. Soc Person Psychol Compass. 2011; 5:976-988. 

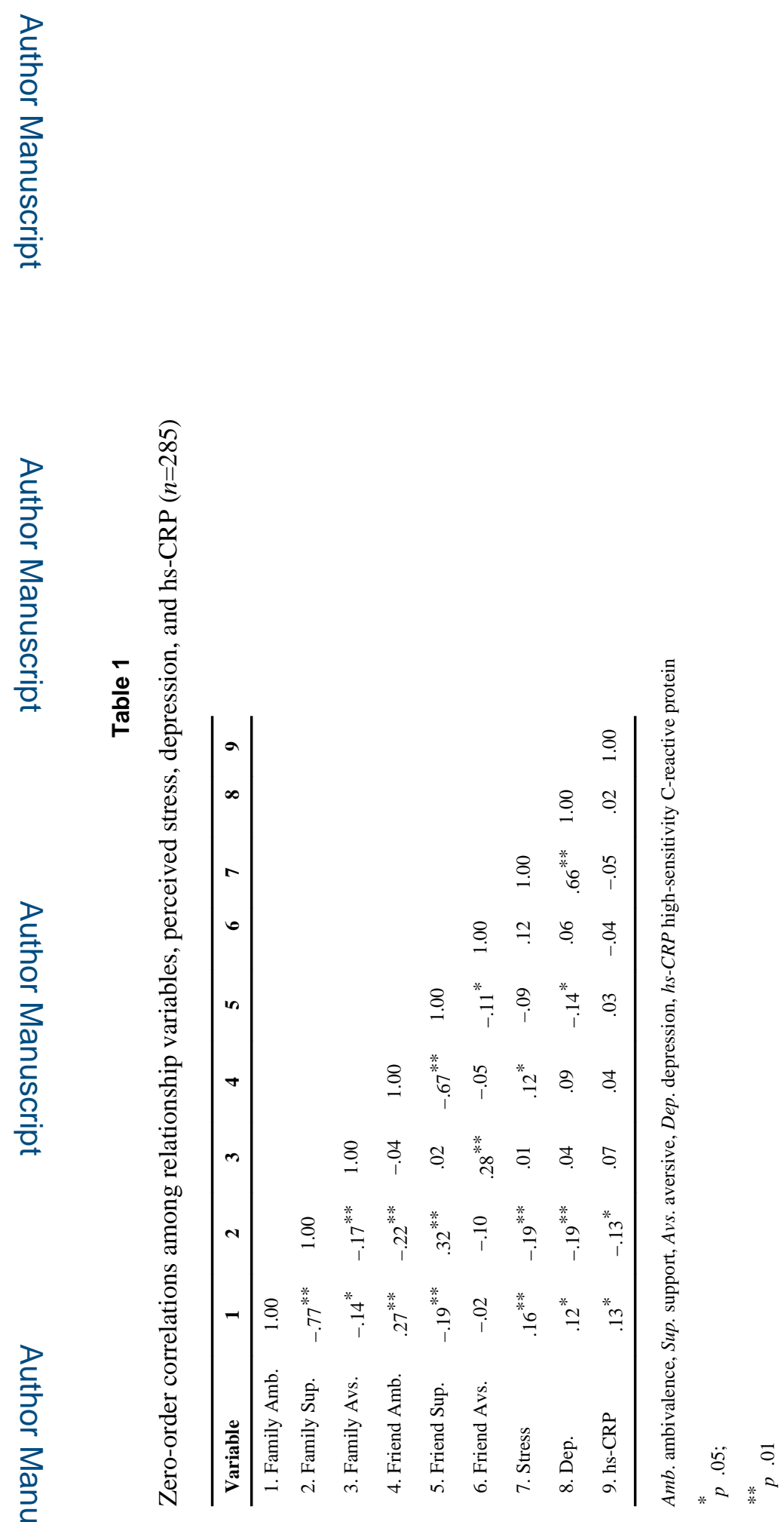

Ann Behav Med. Author manuscript; available in PMC 2016 October 01. 


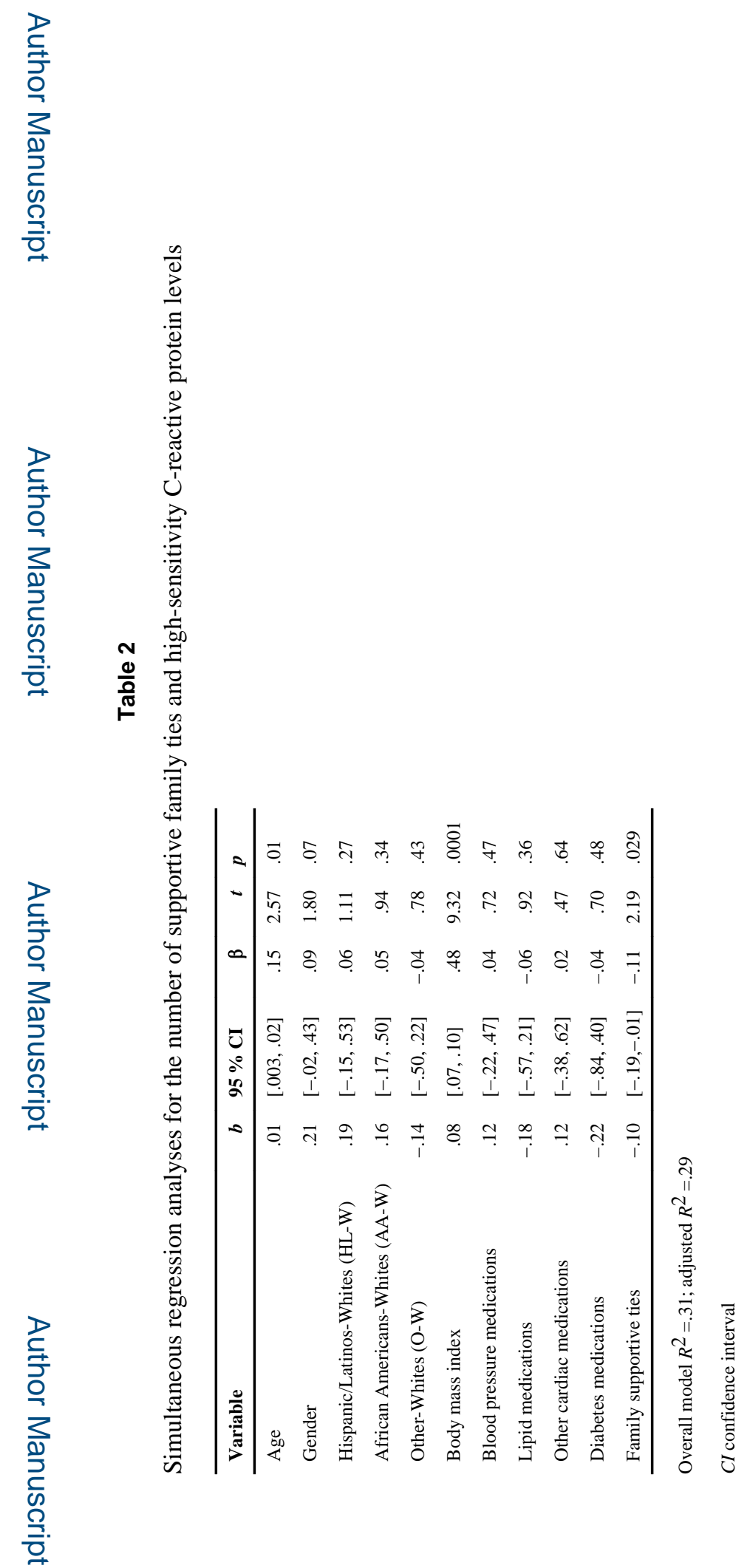

Ann Behav Med. Author manuscript; available in PMC 2016 October 01. 


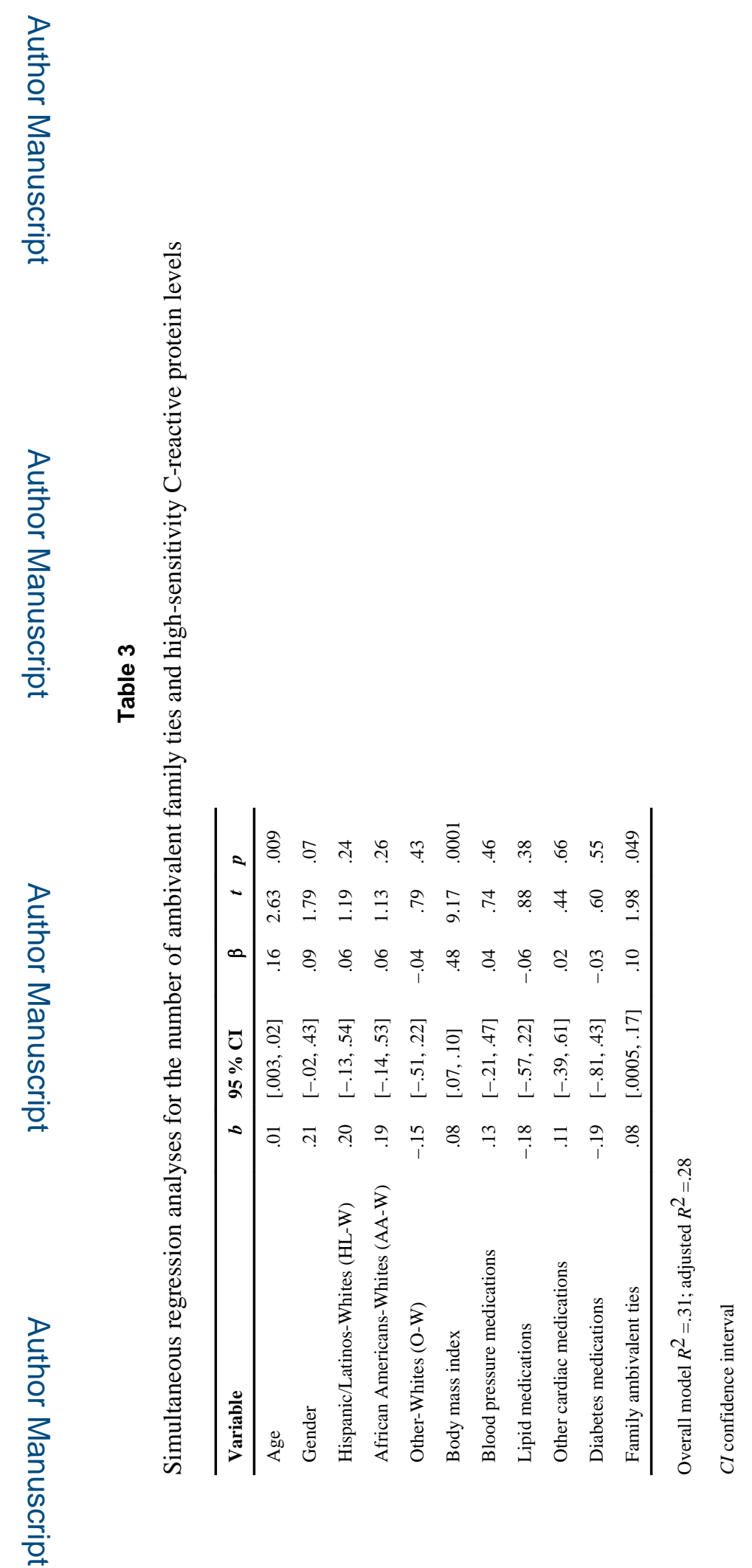

Ann Behav Med. Author manuscript; available in PMC 2016 October 01. 